\title{
Foot Size Assessment in Children with Congenital Talipes Equinovarus on Bracing Following Ponseti Method
}

\author{
Debasish Meher ${ }^{1}$, Saurabh Kumar ${ }^{2}$, Aditya N Aggarwal ${ }^{3}$, Rajnand Kumar ${ }^{4}$
}

\begin{abstract}
Introduction: Foot length (FL) and width (FW) were measured in unilateral idiopathic congenital talipes equinovarus (CTEV) treated via the Ponseti method and difference was compared in foot sizes, if any.

Material and methods: Total 30 patients were enrolled in study, comparing FL and FW in affected vs unaffected in unilateral clubfeet and analyzed statistically.

Results: The mean FL for affected foot was $11.2433 \mathrm{~cm}$ and $11.8380 \mathrm{~cm}$ for unaffected foot. The mean FW for affected foot was $5.5433 \mathrm{~cm}$ and $5.5867 \mathrm{~cm}$ for unaffected foot. The difference between the FL and FW was assessed by the paired $t$ test. The mean FL difference between the affected and unaffected foot was found to be 0.05600 and a $p$ value of 0.716 was found to be statistically insignificant. Likewise, the mean FW difference was found to be 0.0233 and a $p$ value of 0.742 was also statistically insignificant. Both the size difference was statistically insignificant and was comparable to each other.

Conclusion: Foot length and FW between the affected and the unaffected foot are comparable to each other and the mean difference between these two is statistically insignificant.

Keywords: Clubfeet, Congenital talipes equinovarus, Foot length, Foot width.

Journal of Foot and Ankle Surgery (Asia Pacific) (2021): 10.5005/jp-journals-10040-1142
\end{abstract}

\section{INTRODUCTION}

The Ponseti method for clubfoot is gold standard treatment at many orthopedic centers. Various studies on the Ponseti method have demonstrated good to excellent outcome of affected feet using clinical, radiological, functional, gait, pedobarographic, and electrogoniometric analyzes. ${ }^{1}$

The result of clubfoot treatment depends on various criteria but these scoring systems neglect the dimensions of foot length (FL) and foot width (FW), which are responsible for cosmesis and footwear. $^{2-4}$

Normal foot size is not achieved by the Ponseti method of treatment but Fulton et al. suggested that it achieves a near normal anatomic foot as compared to surgical treatment. ${ }^{5}$

The newer generation is worried about the foot cosmesis and size in long-term besides adequate functional results. The literature on clubfoot treatment still describes the affected foot to be on average 1-11/2 size smaller when extensive surgeries were the predominant modality for clubfoot treatment. The literature is scanty for FL and FW after the Ponseti method of treatment in unilateral idiopathic clubfoot. ${ }^{1,6,7}$ We measured the FL and FW in Ponseti-treated idiopathic unilateral clubfoot and compared difference in foot sizes if any in children who were on brace following Ponseti casting.

\section{Materials and Methods}

The cross-sectional study was conducted in the orthopedics department at a tertiary care center, Delhi, after ethical committee clearance in 30 children less than 4 years from October 2017 to April 2019 affected with unilateral clubfoot treated with the Ponseti method and are on braces in clubfoot clinic. A consent was taken from parents prior to start of treatment. Children with idiopathic unilateral congenital talipes equinovarus (CTEV) treated by the

\footnotetext{
${ }^{1-4}$ Department of Orthopaedics, University College of Medical Sciences and Guru Teg Bahadur Hospital, Delhi, India
}

Corresponding Author: Saurabh Kumar, Department of Orthopaedics, University College of Medical Sciences and Guru Teg Bahadur Hospital, Delhi, India, Phone: +91 9013211210, e-mail: srbrai@gmail.com

How to cite this article: Meher D, Kumar S, Aggarwal AN, et al. Foot Size Assessment in Children with Congenital Talipes Equinovarus on Bracing Following Ponseti Method. J Foot Ankle Surg (Asia Pacific) 2021;8(3):141-144.

Source of support: Nil

Conflict of interest: None

Ponseti method and who are on brace treatment, and with Pirani score less than or equal to 1, were included for this study. Children who were surgically intervened for clubfoot except tendoachilles lengthening, secondary clubfoot, and parents/care providers refusing to give consent to participate in the study were excluded from the study. All the demographic essentials - name, age, sex side of deformed foot, and duration of bracing - were recorded and all the observations were recorded in a predesigned case record form. The measurement of foot size (FL and FW) was based on the anthropometric method described by Kesemenli et al. ${ }^{3}$ The foot size was measured by keeping the bilateral child's foot on a white paper sheet and marking the outline (Fig. 1). The drawings of both feet were made on A-4 size white paper; FL and FW were measured with millimetric scale (Fig. 2). The measurements were recorded by single orthopedic surgeon in the CTEV clinic.

The FL was measured by taking mean of all the toe lengths measured by taking the distances between the tip of heel and farthest point on each toe.

Toe lengths (Fig. 3): 


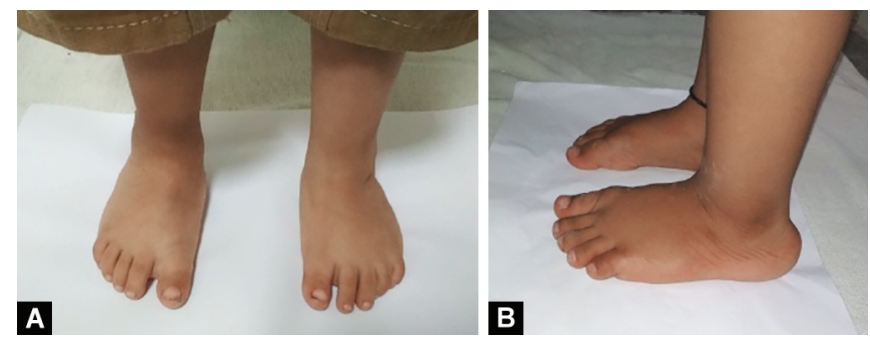

Figs $1 \mathrm{~A}$ and $\mathrm{B}$ : Foot measurements taken by placing the foot on a white paper

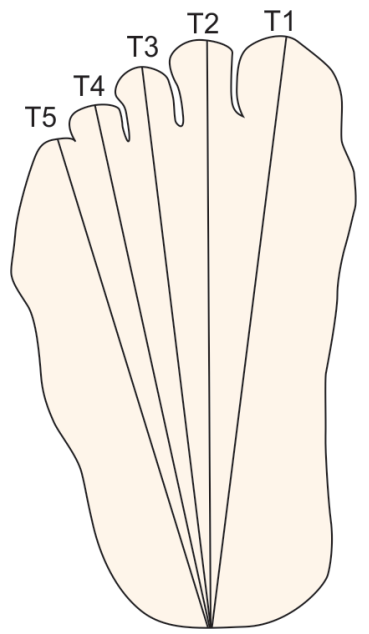

Fig. 3: Measurement of foot size: the distal tip of toe to heel distance was marked on the paper and measured. The mean of the measurements T1-T5 was taken as FL. Distance between the outer margins of the first and fifth tarsometatarsal joints was measured as FW

*T1: distance between the proximal of the calcaneal tubercle (calcaneus) and tip of hallux

*T2: distance between the calcaneus and tip of second toe

*T3: distance between the calcaneus and tip of third toe

*T4: distance between the calcaneus and tip of fourth toe

*T5: distance between the calcaneus and tip of fifth toe

The FW was measured as the distance between the outer margins of the first and fifth tarsometatarsal joints (Fig. 2).

\section{Statistical Analysis}

The measurements were recorded on the Microsoft Excel sheet and the mean difference in FL and FW was assessed by the paired $t$ test on the SPSS software. Standard deviation and standard error of the mean were calculated as well as the $p$ value. The $p$ value of less than 0.05 was taken to be statistically significant.

\section{Observation and Results}

There were 30 children of idiopathic unilateral clubfoot out of which maximum were of $2-3$ years and no patients were above 3 years. The male to female ratio in the series was 3.33:1 (Table 1). The right foot was more commonly involved and the ratio of involvement of the right foot to the left foot was 1.5:1 (Table 2). Foot size was measured at any point of time after the child is treated by Ponseti corrective cast and tenotomy and when the child is on braces. The mean FL for affected foot was $11.2433 \mathrm{~cm}$ and $11.8380 \mathrm{~cm}$ for unaffected foot. The mean FW for affected foot was $5.5433 \mathrm{~cm}$ and 5.5867 $\mathrm{cm}$ for unaffected foot (Table 3). The mean FL difference between
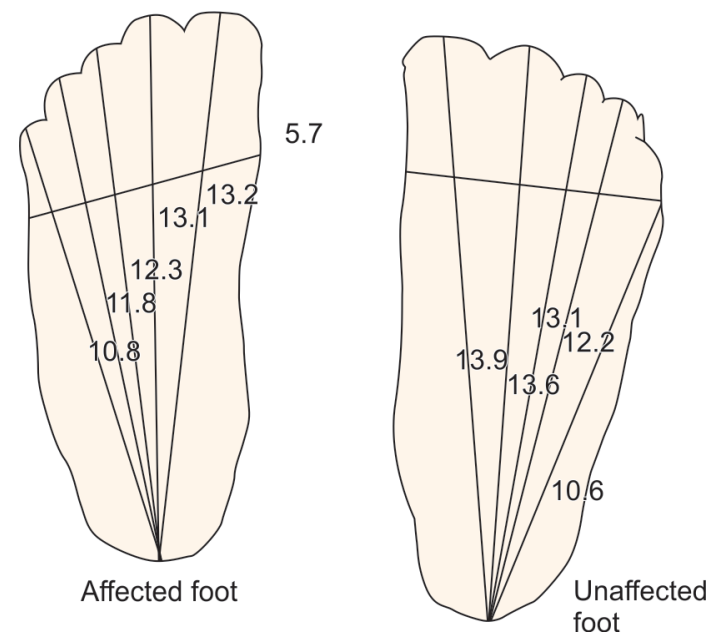

Fig. 2: Foot length and width measurements done in a case

Table 1: Sex distribution

\begin{tabular}{lcccc}
\hline & Frequency & Percentage & $\begin{array}{l}\text { Valid } \\
\text { percent }\end{array}$ & $\begin{array}{l}\text { Cumulative } \\
\text { percent }\end{array}$ \\
\hline Females & 9 & 30 & 30 & 30 \\
Males & 21 & 70 & 70 & 100 \\
Total & 30 & 100 & 100 & \\
\hline
\end{tabular}

Table 2: Foot side distributions

\begin{tabular}{llccc}
\hline & Frequency & Percentage & $\begin{array}{l}\text { Valid } \\
\text { percent }\end{array}$ & $\begin{array}{l}\text { Cumulative } \\
\text { percent }\end{array}$ \\
\hline Left & 12 & 40 & 40 & 40 \\
Right & 18 & 60 & 60 & 100 \\
Total & 30 & 100 & 100 & \\
\hline
\end{tabular}

Table 3: Foot length and width measurement (affected vs unaffected)

\begin{tabular}{lllll}
\hline & Mean & $N$ & $\begin{array}{l}\text { Std. } \\
\text { deviation }\end{array}$ & $\begin{array}{l}\text { Std.error } \\
\text { mean }\end{array}$ \\
\hline Length affected & 11.2433 & 30 & 2.06756 & 0.37748 \\
Length unaffected & 11.8380 & 30 & 1.91359 & 0.34937 \\
Width affected & 5.5433 & 30 & 0.71904 & 0.13128 \\
Width unaffected & 5.5867 & 30 & 0.67402 & 0.12306 \\
\hline
\end{tabular}

the affected and unaffected foot was found to be 0.05600 with a standard deviation of 0.86302 and a $p$ value of 0.716 , which came out to be statistically insignificant. Likewise, the mean FW difference was found to be 0.0233 with a standard deviation of 0.3839 and a $p$ value of 0.742 , which is also statistically insignificant (Table 4).

\section{Discussion}

The goal of clubfoot management is to provide long-term correction of the deformity resulting in a foot that is fully functional, mobile, pain free, without callosities, and able to wear normal shoes. Both surgical and conservative method have resulted in good functional outcome. ${ }^{3,8-11}$ Only few studies have quantified the $\mathrm{FL}$ and $\mathrm{FW}$ of children treated by the Ponseti method of serial casting. ${ }^{1,3,12}$ Kesemenli et al. in their study of 68 children reported 
Foot Size Assessment in Children with Congenital Talipes Equinovarus on Bracing Following Ponseti Method

Table 4: Size difference (affected vs unaffected)

\begin{tabular}{lllllll}
\hline & Mean & Standard deviation & $\begin{array}{l}\text { Standard error } \\
\text { of mean }\end{array}$ & $\begin{array}{l}\text { Lower 95\% } \\
\text { confidence interval }\end{array}$ & $\begin{array}{l}\text { Upper 95\% } \\
\text { confidence interval }\end{array}$ & $p$ value \\
\hline FL difference & 0.05600 & 0.83602 & 0.15264 & -0.25618 & 0.36818 & 0.716 \\
FW difference & 0.02333 & 0.3839 & 0.0701 & -0.1667 & 0.1200 & 0.742 \\
\hline
\end{tabular}

$67.64 \%$ males and $32.35 \%$ females. In a similar study, Agarwal et al. reported 100 patients with a male to female ratio of 3:1, which is similar to our series., ${ }^{1,3}$ Total $18 / 30$ of our cases had right foot involvement and 12/30 of our cases had left foot involvement, which is in concordance to other series. In unilateral cases, a slight preponderance of left side involvement was reported by Agarwal et al. In a study by Kesemenli et al., they did not describe the left or right preponderance., ${ }^{1,3}$ Out of 30 patients included in our study, maximum children were between 2 and 3 years. No patients were below 2 months or above 3 years. Treatment was initiated as soon as the child came to us and braces were put after correction of all the deformities by serial casts. In our study, we found that post correction of unilateral clubfoot via Ponseti method had comparable FL and FW and difference between affected and unaffected foot. Kesemenli et al. included 68 children in their study and divided them into three groups: conservatively treated in unilateral clubfeet; surgically treated clubfoot; and conservatively treated on one side and surgically treated on the other. The first group was compared with our study. The average age in the series was 9 years (range 7-12 years). The conservatively treated foot with follow-up of mean 9 years was on average of $0.91 \mathrm{~cm}(0.4-2.1 \mathrm{~cm})$ shorter in length than the unaffected foot $(p$ $<0.01)$. The differences were statistically significant The FW was shorter by average $0.05 \mathrm{~cm}(0.1-0.4 \mathrm{~cm})(p>0.05)$. The differences were statistically insignificant. But the method utilized for casting was not the Ponseti method. ${ }^{1}$ Another study (2007) by Chesey et al. did comparison between subjective and objective outcome and enrolled $(n=206)$ patients in which 65 patients were having unilateral clubfeet. They concluded that FL correlated with satisfaction but there was no evidence of a statistically significant associated between FW and satisfaction. This study also not used Ponseti as the method of treatment for clubfeet. ${ }^{13}$ Gamble et al. published a prospective longitudinal study comparing children with unilateral clubfeet treated by posterior medial release ( $n$ $=65)$ and the Ponseti method $(n=28)$ with average follow-up duration of 68 months. Mean percentage foot difference was 8.7\% (95\% CL 7.54-9.87\%). ${ }^{12}$ Maffuli et al. did an anthropometric study on 10 boys and 4 girls with total 22 surgically treated feet with radiographic evidence of fusion of ossification center. Foot length was not significantly influenced by the surgery but FW was affected. However, this study was not comparative but was an investigation of whether repeat surgery affected foot dimensions. ${ }^{2}$ Agarwal et al. studied on 100 children of clubfoot who were either unilaterally or bilaterally affected. The author found bilateral feet were similar in size. The unilateral affected feet matched in size with unaffected feet. The size difference between bilateral and unilateral affected feet was not significant. Even the size difference between bilateral and unilateral affected feet was not significant. When they compared overall clubfoot size vs unaffected feet after age matching, a statistical significant difference was apparent (in length, $p=0.03$ and in width, $p=0.02$ ). On the whole, the clubfeet on an average were $0.7 \mathrm{~cm}$ (5.3\%) shorter than the unaffected feet. The bilateral feet were significantly smaller than age-matched unilateral unaffected feet. The length was $0.8 \mathrm{~cm}(6.1 \%)$ shorter than the contralateral feet with a $p$ value of 0.03 and the width was 0.2 $\mathrm{cm}(3.7 \%)$ shorter than the contralateral feet with a $p$ value of 0.03 . ${ }^{1}$ The study also found that Ponseti-managed unilateral foot size was comparable with unaffected foot during the bracing duration and even after the bracing protocol was over, i.e., after 3 years. This point emphasizes the importance of the maintenance phase (a brace protocol of 3-4 years post correction) in the Ponseti method of treatment. The foot size is achieved after the correction phase but it's maintained during bracing and post-bracing period. ${ }^{1}$ Our study was carried out on 30 children with unilateral clubfoot who were corrected by Ponseti casts and achilles tenotomy (if found necessary). On the whole, FL of affected foot was smaller (0.59) and FW was smaller (0.04). The mean FL difference between the affected and unaffected foot was found to be 0.05600 and a $p$ value of 0.716 , which came out to be statistically insignificant. Likewise the mean FW difference was found to be 0.0233 and a $p$ value of 0.742 , which is also statistically insignificant. These results were similar to the study by Agarwal et al. ${ }^{1}$ However, the limitation of the current study is a relatively small sample size. A larger multicentric study must be carried out.

\section{Conclusion}

Foot lengths between the affected and the unaffected foot in unilateral clubfoot are comparable to each other and the mean difference between these two is statistically insignificant. Foot widths between the affected and unaffected foot are comparable to each other and the mean difference between these two is statistically insignificant.

\section{References}

1. Agarwal A, Rastogi A. Anthropometric measurements in Ponseti treated clubfeet. SICOT J 2018;4:19. DOI: 10.1051/sicotj/2018010.

2. Maffulli N, Kenward MG, Irwin AS, et al. Assessment of late results of surgery in talipes equino-varus: a reliability study. Eur J Paediatr 1997;156(4):317-319. DOI: 10.1007/s004310050608.

3. Kesemenli CC, Kapukaya A, Subaşi M, et al. Anthropometric study of patients treated for clubfoot. J Pediatr Orthop 2003;23(4):498-502. DOI: 10.1097/01241398-200307000-00016.

4. Davies TC, Kiefer G, Zernicke RF. Kinematics and kinetics of the hip, knee, and ankle of children with clubfoot after posteromedial release. J Pediatr Orthop 2001;21(3):366-371. DOI: 10.1097/01241398200105000-00020.

5. Fulton Z, Briggs D, Silva S, et al. Calf circumference discrepancies in patients with unilateral clubfoot: Ponseti vs surgical release. J Pediatr Orthop 2015;35(4):403-406. DOI: 10.1097/BPO.0000000000000277.

6. Club foot. Wikipedia. https://en.wikipedia.,org/wiki/Club_foot (Assessed: 2019/20/04).

7. Cooper DM, Dietz FR. Treatment of idiopathic clubfoot. J Bone Joint Surg 1995;77(10):1477-1489. DOI: 10.2106/00004623-19951000000002.

8. Jain AK, Zulfiqar AM, Kumar S. Evaluation of foot bimalleolar angle in the management of congenital talipes equinovarus. J Pediatr Orthop 2001;21(1):55-59. DOI: 10.1097/01241398-200101000-00012. 
9. Kapukya A, Subasi M, Kesemenli C, et al. Complete subtalar release in the surgical treatment of congenital peseqinovarus. Hacettepe $J$ Orthop Surg 1999;9:57-61.

10. Karol LA, Concha MC, Johnston 2nd CE. Gait analysis and muscle strength in children with surgically treated clubfeet. J Pediatr Orthop 1997;17(6):790-795. DOI: 10.1097/01241398-199711000-00018.

11. Roye BD, Vitale MG, Gelijns AC, et al. Patient-based outcomes after clubfoot surgery. J Pediatr Orthop 2001;21(1):42-49. DOI: 10.1097/01241398-200101000-00010.
12. Gamble J, Batista E, Rinsky L. How small is the leg and foot in unilateral clubfoot. Orthop Proc 2018;94-B(SuppI XXI):Available from http:// bjjprocs.boneandjoint.org.uk/content/94-B/SUPP_XXI/34. Accessed on 21 April 2019.

13. Chesney D, Barker S, Maffulli N. Subjective and objective outcome in congenital clubfoot: a comparative study of 204 children. BMC Musculoskelet Disord 2007;8:53. DOI: 10.1186/14712474-8-53. 